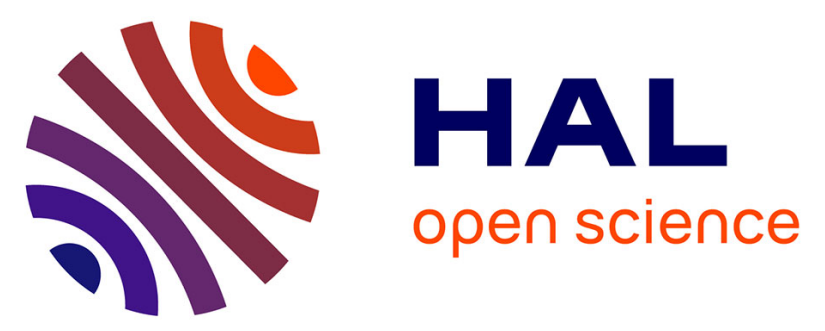

\title{
Polyunsaturated fatty acids inhibit PI3K activity in a yeast-based model system.
}

Elodie Couplan, Christelle Le Foll, Marie Le Cann, Charlotte Corporeau, Marc Blondel, Jacques Delarue

\section{- To cite this version:}

Elodie Couplan, Christelle Le Foll, Marie Le Cann, Charlotte Corporeau, Marc Blondel, et al.. Polyunsaturated fatty acids inhibit PI3K activity in a yeast-based model system.. Biotechnology Journal, 2009, 4 (8), pp.1190-n/a. 10.1002/biot.200800229 . hal-00495059

\section{HAL Id: hal-00495059 https://hal.science/hal-00495059}

Submitted on 25 Jun 2010

HAL is a multi-disciplinary open access archive for the deposit and dissemination of scientific research documents, whether they are published or not. The documents may come from teaching and research institutions in France or abroad, or from public or private research centers.
L'archive ouverte pluridisciplinaire HAL, est destinée au dépôt et à la diffusion de documents scientifiques de niveau recherche, publiés ou non, émanant des établissements d'enseignement et de recherche français ou étrangers, des laboratoires publics ou privés. 


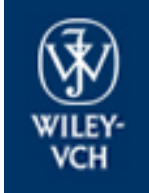

Biotechnology Journal

A yeast-based assay shows that long chain $\mathbf{n}-\mathbf{3}$ polyunsaturated fatty acids and arachidonic acid inhibit PI3K activity in vivo

\begin{tabular}{|c|c|}
\hline Journal: & Biotechnology Journal \\
\hline Manuscript ID: & BIOT-2008-0229.R1 \\
\hline Wiley - Manuscript type: & Research Article \\
\hline $\begin{array}{r}\text { Date Submitted by the } \\
\text { Author: }\end{array}$ & 02-Apr-2009 \\
\hline Complete List of Authors: & $\begin{array}{l}\text { Couplan, Elodie; Faculté de Médecine CHU de Brest, INSERM U613 } \\
\text { Le Foll, Christelle; Faculté de Médecine, UBO } \\
\text { Le Cann, Marie; Faculté de Médecine CHU de Brest, INSERM U613 } \\
\text { Corporeau, Charlotte; IFREMER } \\
\text { Blondel, Marc; INSERM U613, Faculte de Médecine CHU de Brest } \\
\text { Delarue, Jacques; Faculté de Médecine, UBO }\end{array}$ \\
\hline Keywords: & polyunsaturated fatty acids, PI3K inhibition, nutrition, cancer \\
\hline
\end{tabular}

\section{ScholarONE \\ Manuscript Central}


Couplan et al.

\section{A yeast-based assay shows that long chain n-3 polyunsaturated fatty acids and arachidonic acid inhibit PI3K activity in vivo}

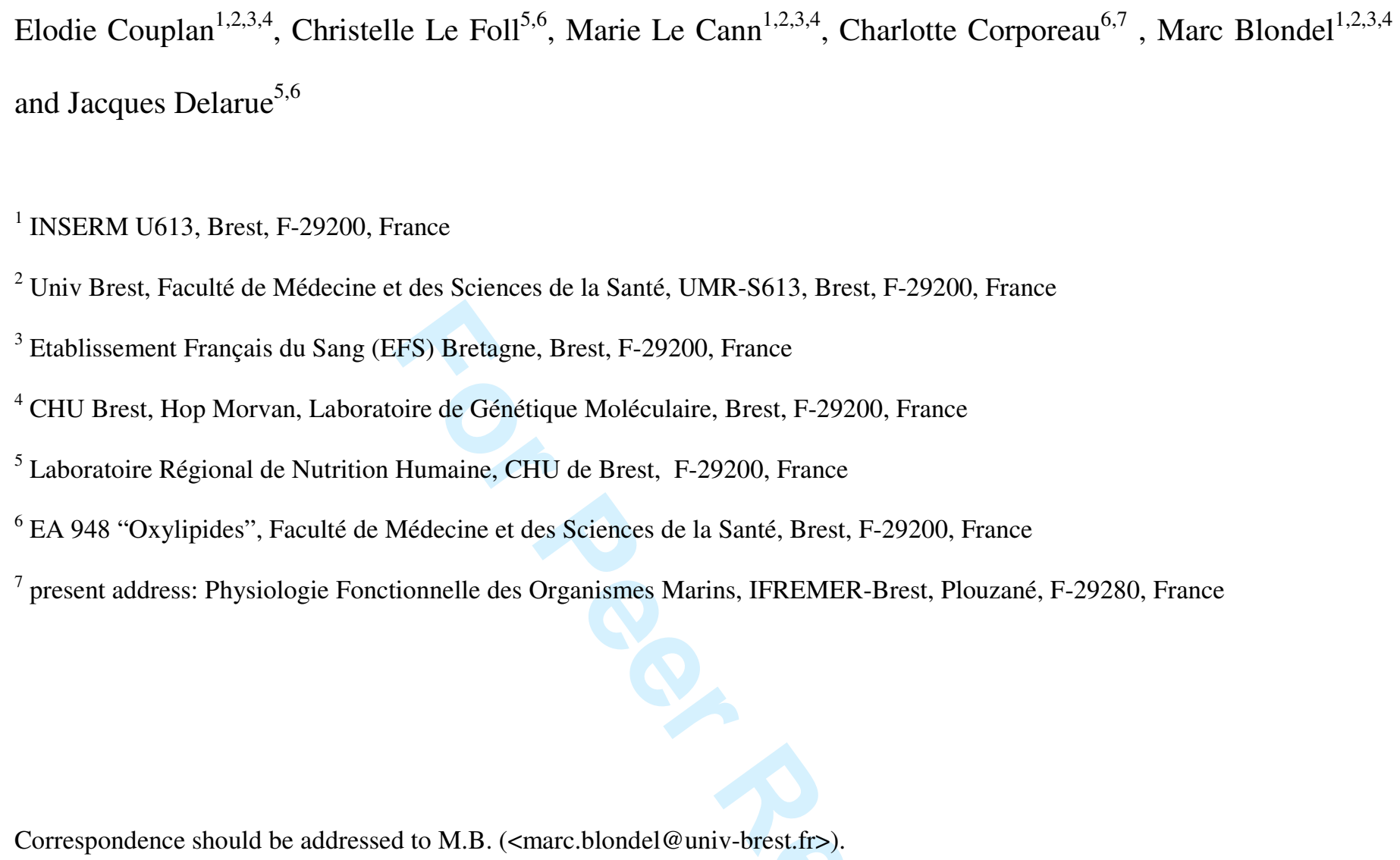

Abbreviations used: AA, arachidonic acid; DHA, docosahexaenoic acid; EPA, eicosapentaenoic acid; PDK1, 3'phosphoinositide-dependent kinase 1; PH, pleckstrin homology; PI3K, phosphatidyl inositol 3' kinase; PI, phospatidyl inositol; PIP, phosphatidyl inositol 3-phosphate; PIP $_{2}$, phosphatidyl inositol 4,5-bisphosphate; $\mathbf{P I P}_{3}$, phosphatidyl inositol 3,4,5trisphosphate; PKB, protein kinase B; PLC $\boldsymbol{\delta} 1$, phospholipase $\delta 1$; PUFA, polyunsaturated fatty acids; PTEN, phosphatidylinositol 3-phosphatase; SH2, Src homology 


\section{ABSTRACT}

The PI3K (phosphatidyl inositol 3' kinase) pathway controls the regulation of cell growth, proliferation, migration and apoptosis. In many tumors, the PI3K gene is mutated or overexpressed, and/or the PI3K pathway is hyperactive. PI3K is therefore a potential pharmacological target for the development of anti-tumor drugs. Some polyunsaturated fatty acids (PUFA), when given in the diet, may lead to a decrease in PI3K activity. We used a yeast-based model to reconstitute the PI3K/PTEN/Akt pathway to study the effects of long chain n-3 fatty acids on PI3K kinase and found that various PUFA were able to alleviate toxicity induced by overexpression of PI3K. The various PUFA had no significant effect on the steady-state level of PI3K catalytic subunit proteins in yeast. However, depletion of $\mathrm{PIP}_{2}$ due to overexpression of the p110a subunit was significantly reduced by treating the yeast cells with the various PUFA. The inhibition of mammalian PI3K, expressed in an exogenous cellular context in yeast, is likely to be a direct effect of these PUFA on PI3K rather than on other mammalian endogenous or environmental factors. These results are particularly promising given the abundance of active PUFA in marine foodstuffs and especially fish oils.

\section{INTRODUCTION}

The phosphatidylinositol 3'-kinase (PI3K) signaling pathway controls the regulation of cell growth, proliferation, migration and apoptosis $[1,2]$. Type $1 \mathrm{~A}$ PI3K is composed of a regulatory subunit, p85, which is responsible for its plasma membrane localization and a catalytic subunit, p110, responsible for its kinase activity. The activation of the catalytic subunit, p110, targets PI3K to its substrate phosphatidyl inositol 4,5biphosphate $\left(\mathrm{PIP}_{2}\right)$ localized at the plasma membrane. The activity of p110 generates phosphatidyl inositol 3,4,5-trisphosphate $\left(\mathrm{PIP}_{3}\right)$ from $\mathrm{PIP}_{2}$ in the plasma membrane. $\mathrm{PIP}_{3}$ in turn generates recognition sites for proteins containing a plekstrin homology (PH) domain; these proteins include 3'-phosphoinositidedependent kinase 1 (PDK1) and protein kinase B (PKB/Akt) which both translocate to the inner surface of the plasma membrane in response to the generation $\mathrm{PIP}_{3}$. Once at the surface of the plasma membrane, PKB/Akt is phosphorylated on Thr308 by PDK1 and fully activated by phosphorylation on Ser473 by PDK2 
kinases (mainly mTOR complex 2 and DNA-PKcs). Once activated, PKB/Akt phosphorylates a myriad of proteins which control cell growth, cell proliferation and apoptosis, all steps involved in tumor formation and malignant cell dissemination [2]. The PI3K pathway is negatively regulated by two phosphatases: SHIP (SH2 domain-containing inositol phosphatase) and PTEN (phosphatase and tensin homolog deleted in chromosome ten). In many tumors, the PI3K gene is mutated or overexpressed, and PTEN function is attenuated or the protein underexpressed; consequently, the PI3K/Akt pathway is overactive [3]. Thus, PI3K is involved in the development of many types of cancers $[4,5]$. It also seems to be involved in the radioresistance mechanism of head and neck cancers [6]. Indeed, generation of $\mathrm{PIP}_{3}$ at the plasma membrane is pivotal for the regulation of mammalian cell proliferation and survival by growth-promoting factors [1]. Therefore, PI3K is an attractive pharmacological target for screening for anti-cancer drugs [3].

Most known inhibitors of PI3K are synthetic molecules [3], but there is evidence that long chain n-3 fatty acids (i.e. EPA and DHA) found in marine foodstuffs, especially fish oils, may inhibit PI3K activity in vivo. Indeed, long chain n-3 fatty acids act as protein kinase inhibitors in the central nervous system [7]. In rats consuming a small amount of fish oils (2.2\% of calories) in a normolipidic diet, PI3K activity in both liver and muscle is 54\% lower than that in rats on a control diet without fish oil, as judged by determining in vitro the activity of PI3K after immuno-precipitation from cell extracts [8]. Also, EPA restores tamoxifen sensitivity to breast cancer cells with high Akt activity [9]. Here, we confirm that long chain n-3 fatty acids are inhibitors of PI3K activity. We used, as a model system, the reconstitution of the mammalian PI3K/PTEN/Akt pathway in yeast, as described by Rodriguez-Escudero et al [10]. In this model, the catalytic subunit of mammalian PI3K, p110 , is overproduced and artificially targeted to the plasma membrane, where it converts $\mathrm{PIP}_{2}$ to $\mathrm{PIP}_{3}$ : this inhibits cell growth by depleting the cellular pool of $\mathrm{PIP}_{2}$ which is essential for yeast. This effect is completely prevented by the co-expression of catalytically active mammalian PTEN which dephosphorylates PIP $_{3}$. The inhibitory effect on yeast growth is partially relieved by LY294002, a PI3K inhibitor, and similar overproduction of a catalytically inactive mutant of p110 $\alpha$ $\left(\mathrm{p} 110 \alpha^{\mathrm{K} 802 \mathrm{R}}\right)$ has no effect on growth and viability or on $\mathrm{PIP}_{2}$ depletion: these two observations confirm that the inhibitory effect depends on PI3K kinase activity. The production of $\mathrm{PIP}_{3}$ in yeast by induction of the $\mathrm{p} 110 \alpha$ gene induces the translocation of PKB/Akt to the plasma membrane and enhances its 
phosphorylation, as observed in mammals in vivo. Therefore, this model can be used to determine whether EPA and DHA in the culture medium of yeast inhibit the production or catalytic activity or both of the p110 $\alpha$ subunit and this, by freeing itself from any other mammalian endogenous or environmental factors which may interfere in vivo with the effects of long chain n-3 Poly Unsaturated Fatty Acids (PUFA) on PI3K activity. In addition, and contrary to the rat model, in the yeast-based model PI3K activity is directly correlated to in vivo measurable phenotypes.

\section{MATERIALS AND METHODS}

\section{Yeast strains, culture media and plasmids}

The Saccharomyces cerevisiae strains used were the Wild Type (WT) K699: Mata, ade2-1, trp1-1, can1100, leu2-3,112, his3-11,15, ura3, GAL+, psi+, ssd1-d2 (W303 genetic background) and its hoflA derivative: Mata, ade2-1, trp1-1, can1-100, leu2-3,112, his3-11,15, ura3, GAL+, psi+, ssd1-d2, hofl::KANMX [11]. YPD (1\% (w/v) yeast extract, $2 \%(\mathrm{w} / \mathrm{v})$ peptone and $2 \%(\mathrm{w} / \mathrm{v})$ glucose) was the general non selective medium used for yeast cell growth. Synthetic minimal medium contained $0.17 \%$ yeast nitrogen base without amino acids, $0.5 \%$ ammonium sulfate, $2 \%$ glucose, $2 \%$ raffinose or $2 \%$ raffinose/ $2 \%$ galactose and lacked appropriate amino acids and nucleic acid bases to maintain selection for plasmids. Solid media contained 2\%(w/v) agar. Yeast transformations were performed by the lithium acetate procedure [12]. The plasmids YCpLG-PI3K and YCpLG-PI3K ${ }^{\mathrm{K} 802 \mathrm{R}}$, allowing expression of $\mathrm{p} 110 \alpha$ and $\mathrm{p} 110 \alpha^{\mathrm{K} 802 \mathrm{R}}$, the catalytic subunit of a membrane-targeted mammalian PI3K, under the control of the galactose-inducible GAL1 promoter were described in [10]. The plasmids pBM328, allowing expression of hof $1 \Delta \mathrm{PEST}$ (under the control of the inducible $G A L 1,10$ promoter), and the empty vector, pRD53, were described previously [11]. To visualize $\mathrm{PIP}_{2}$ in vivo, the plasmid pRS426-GFP-2xPH (PLC $\delta$ ) which allows expression of the GFP protein fused to two tandem copies of the PLC $\delta 1$ PH domain was used [10, 13].

\section{Yeast-based assay}

This assay was adapted from an existing test $[14,15]$. Briefly, $240 \mu 1$ of a culture of exponentially growing cells, adjusted to an $\mathrm{OD}_{600}$ of 0.25 , were spread homogeneously with sterile glass beads $(\approx 3 \mathrm{~mm}$ diameter $)$ 
on a square Petri dish $(12 \mathrm{~cm} \times 12 \mathrm{~cm})$ containing synthetic minimal medium and $2 \%$ glucose or $2 \%$ raffinose and $2 \%$ galactose. Sterile filters (similar to those used for antibiograms) were placed on the agar surface. A volume of $1 \mu 1$ of a pure solution of the fatty acids (DHA, EPA, AA or oleic acid, as indicated) was spotted onto the filters as indicated in the left panel of Figures 2 and 3 . Aliquots of $1 \mu$ of bromopalmitate or LYS294002 (PI3K inhibitor) in solution in DMSO (at $200 \mu \mathrm{M}$ and $100 \mu \mathrm{M}$, respectively) were similarly tested on the same Petri plates. As a negative control, a filter was loaded with $1 \mu$ l of DMSO, the vehicle. Plates were then incubated at $30^{\circ} \mathrm{C}$ for three to five days and scanned using a Snap Scan 1212 (Agfa).

\section{Growth curves and protein detection}

Exponentially growing cells were cultured in a liquid minimal selective medium containing $2 \%$ raffinose overnight at $30^{\circ} \mathrm{C}$, diluted and allowed a few hours of recovery in the same medium. Then, $2 \%$ galactose with, where indicated, $0.005 \%$ of DHA, EPA, AA (from a 5\% solution in DMSO) or DMSO was added to the yeast cultures. For growth curves, $\mathrm{OD}_{600 \mathrm{~nm}}$ was determined at the times indicated. For protein detection, 3 to $5 \mathrm{ml}$ of yeast cultures were incubated for six hours $\left(\mathrm{OD}_{600}\right.$ around 0.5$)$ then rapidly harvested by centrifugation. The cell pellets were cooled on ice, resuspended in $150 \mu 1$ of cold lysis buffer $(1.85 \mathrm{M} \mathrm{NaOH}$, $13 \% \beta$-mercaptoethanol) and $150 \mu \mathrm{l}$ of a cold TCA solution at $50 \%$ was added. The samples were centrifuged at $15,000 \mathrm{~g}$ for 2 minutes at $4^{\circ} \mathrm{C}$ : the precipitated proteins were washed with $1 \mathrm{ml}$ of cold acetone and centrifuged again. The acetone was discarded, Laemmli buffer added and the samples were heated at $95^{\circ} \mathrm{C}$ for $3 \mathrm{~min}$. These protein extracts were then analyzed by SDS-PAGE and transferred to a nitrocellulose membrane (Schleicher \& Schuell). Membranes were incubated with primary antibody (at 1:1,000 for antiPI3K p110 $\alpha$, Cell Signaling Technology or 1:10,000 for anti-actin, Calbiochem JLA20) in TBST buffer (50mM Tris, $\mathrm{pH} 7.4,150 \mathrm{mM} \mathrm{NaCl}, 0.1 \%$ Tween 20 ) containing 5\% non-fat dry milk for 1 hour. The membrane were then washed with TBST, incubated with secondary antibody (1:3,000 goat anti-rabbit antibody conjugated to horseradish peroxidase, Biorad, for anti-PI3K p110 $\alpha$ and 1:2,000 goat anti-mouse IgM, Calbiochem, for anti-actin). Enhanced Chemiluminescence (ECL, Amersham) and a Vilbert-Lourmat Photodocumentation Chemistart 5000 imager were used to reveal bound antibody. 


\section{Microscopy}

Cells co-transformed with the plasmids YCpLG-PI3K or YCpLG-PI3K ${ }^{\mathrm{K} 802 \mathrm{R}}$ and the pRS426-GFP2xPH(PLC 81$)$ vector (which allows constitutive expression of GFP-PH) were grown overnight in a liquid minimal selective medium containing raffinose. Cultures were diluted and allowed to recover for a few hours in the same medium, and $2 \%$ galactose (to induce PI3K overexpression) or $2 \%$ glucose (to repress PI3K) was added with $0.005 \%$ of DHA, EPA, AA or DMSO (from a 5\% solution in DMSO). The GFP signal was visualized after 8 hours using a Chroma GFP4 filter (excitation 455-495 nm) on an Olympus BX61 microscope, photographed with a Spot RT cooled CCD camera and analyzed with Photoshop 7.0 software (Adobe). To obtain reliable and statistically significant data, $\geq 250$ cells were examined for each condition. Values reported are means of three independent experiments.

\section{RESULTS}

\section{Ability of fatty acids EPA, DHA and AA to alleviate PI3K-driven toxicity in yeast}

The mammalian PI3K/PTEN/Akt pathway has been reconstituted in the yeast $S$. cerevisiae in which PI3K expression and activity inhibits cell growth [10]. Expression of the p110 $\alpha$ catalytic subunit of PI3K under the control of the strong galactose-inducible GAL1 promoter led to the conversion of the essential $\mathrm{PIP}_{2}$ pool into $\mathrm{PIP}_{3}$. This $\mathrm{PI} 3 \mathrm{~K}$ overexpression-dependent depletion of $\mathrm{PIP}_{2}$ impaired yeast growth by altering morphogenesis and vesicular trafficking (Figure 1, upper panel and [10]). An analogous construct was obtained with an allele of PI3K carrying a point mutation $\left(\mathrm{Lys}^{802} \rightarrow \mathrm{Arg}\right.$ ) which encodes a catalytically inactive subunit (p110 $\alpha^{\mathrm{K} 802 \mathrm{R}}$ ) (Figure 1, lower panel): there was no growth inhibition confirming that the effect was due to the kinase function of PI3K [10]. In both the $\mathrm{p} 110 \alpha$ and $\mathrm{p} 110 \alpha^{\mathrm{K} 802 \mathrm{R}}$ constructs, the coding regions were fused in frame at their 3' end with the coding sequence of the CAAX box of H-ras to target the subunits to the plasma membrane. This was to compensate for the absence of p85, the regulatory subunit of $\mathrm{PI} 3 \mathrm{~K}$, which is responsible for the plasma membrane localization of the enzyme in mammals. Therefore the expressed $\mathrm{p} 110 \alpha$ can be considered as a constitutively active form of PI3K. 
Couplan et al.

Using the same expression vectors as those used by Rodriguez-Escudero et al. who developed this model, $\mathrm{p} 110 \alpha$ and $\mathrm{p} 110 \alpha^{\mathrm{K} 802 \mathrm{R}}$ were overproduced in S. cerevisiae under the control of the strong galactoseinducible GAL1 promoter. We then tested the ability of various PUFA to suppress PI3K overexpressiondependent growth inhibition. Wild-type (WT) S. cerevisiae cells (K699) transformed with the vectors were spread on minimal synthetic medium under either repressing (glucose medium) or inducing conditions (galactose medium). Sterile filters were then placed on the agar surface and various fatty acids were deposited onto the filters as indicated in Figure 2, left panel. This simple method allows creation of a full gradient of concentration of a compound around the filter where it was deposited $[14,15]$. We tested two n-3 PUFA found in fish oils, docosahexaenoic acid (DHA, C22:6 n-3) and eicosapentaenoic acid (EPA, C20:5 n-3), and arachidonic acid (AA, C20:4 n-6), an n-6 PUFA which presents the same carbon chain length as EPA. We also evaluated the effects of oleate (or oleic acid), a monounsaturated n-9 fatty acid; bromopalmitate, a non- metabolizable fatty acid (an inhibitor of a variety of enzymes and proteins associated with lipid metabolism) and DMSO, the vehicle used to dissolve some of the molecules tested. The PI3K inhibitor, LY294002, which restores cell growth to yeast overproducing PI3K [10], was used as a positive control. After three days at $30^{\circ} \mathrm{C}$ and as previously observed [10], $W T$ yeast cells expressing p110 $\alpha$ failed to grow on galactose medium whereas $W T$ yeast expressing $\mathrm{p} 110 \alpha^{\mathrm{K} 802 \mathrm{R}}$, the catalytically inactive PI3K, grew normally (Figure 2). Both grew normally on glucose medium on which the expression of both forms of p110 $\alpha$ is repressed. DHA and EPA (the two n-3 PUFA), and AA (the n-6 PUFA), all relieved the growth inhibition, as judged by the appearance of a growth halo around the filters on which they were deposited. Oleate, bromopalmitate and DMSO had no such effect on yeast growth. Thus, like the PI3K kinase inhibitor LY294002, both n-3 and n-6 PUFA were able to alleviate PI3K-induced growth inhibition in yeast, suggesting that they may inhibit the kinase activity of PI3K in vivo. Interestingly, no clear synergistic effect was observed between the various PUFA on one hand and LY294002 on the other hand (data not shown and Figure 2), suggesting that they act via a common mechanism.

The ability of AA, EPA and DHA to alleviate PI3K-driven toxicity in yeast is not due to an effect on PI3K expression 
There are at least two mechanisms by which AA, EPA and DHA may alleviate yeast growth inhibition due to overexpression of PI3K: a direct or indirect effect on PI3K kinase activity; or interference with PI3K gene expression (for example by affecting transcription from the galactose-inducible GAL1 promoter). If AA, EPA and DHA affect expression of the gene, the abundance of PI3K in yeast would be affected; if the activity of the enzyme is affected, its abundance should be unaffected.

We investigated the effects of AA, EPA and DHA on GAL1 promoter-driven expression by testing the effect of these polyunsaturated fatty acids on the toxicity of hof $1 \triangle \mathrm{PEST}$ overexpressed from this promoter in yeast. hof $1 \triangle \mathrm{PEST}$ is a stabilized truncated form of Hof1, a protein involved in cytokinesis in S. cerevisiae which has to be directed by the E3-ligase $\mathrm{SCF}^{\mathrm{Grr1}}$ to ubiquitin-26S proteasome dependent degradation at the end of mitosis to allow cell separation [11]. Therefore GAL1 promoter-driven overexpression of Hof1, and especially of its stabilized form hof $1 \triangle \mathrm{PEST}$, inhibits yeast cell growth by preventing cell separation at the end of mitosis [11]. We used an experiment similar to that described in Figure 2 except that yeast cells (K699 hof14) were transformed with either a plasmid allowing strong overexpression of hof1 $\mathrm{PEST}$ from the same GAL1 promoter or with an empty vector (negative control). None of AA, EPA or DHA (or DMSO, bromopalmitate, oleate or LY294002) was able to alleviate the growth inhibition due to hof $1 \Delta \mathrm{PEST}$ overproduction. As the only common point between hof1 $\triangle$ PEST-and p110 $\alpha$-driven toxicity is the strong overexpression from the galactose-inducible GAL1 promoter, these results suggest that none of these PUFA significantly interfered with this overexpression (Figure 3a). Next, we tested the effects of AA, EPA and DHA on the steady-state level of $\mathrm{p} 110 \alpha$. Yeast cells carrying $\mathrm{p} 110 \alpha$ under the GAL1 promoter were grown in a galactose medium in the presence of various PUFA (as indicated in Figure 3b). Protein extracts were prepared and analyzed by Western blotting. The amount of p110 $\alpha$ did not differ between cells grown in the presence of AA, EPA and DHA and controls. Findings were similar for yeast cells overexpressing $\mathrm{p} 110 \alpha^{\mathrm{K} 802 \mathrm{R}}$, the catalytically inactive PI3K (data not shown). Using the same cells and under the same conditions, we found that long chain n-3 PUFA and AA also improve the growth in liquid culture of yeast cells overexpressing p110 $\alpha$ (Figure 3c for DHA and data not shown). Therefore, AA, EPA and DHA all appear to act by inhibiting the kinase activity of PI3K. 
Couplan et al.

Inhibition of PI3K by fatty acids

\section{AA, EPA and DHA lead to a partial suppression of PIP 2 depletion}

To confirm that AA, EPA and DHA inhibit the activity of mammalian PI3K kinase in yeast, we studied the effects of these fatty acids on $\mathrm{PIP}_{3}$ production driven by the overexpression p110 $\alpha$. We used $\mathrm{PIP}_{2}$ depletion as a marker of the kinase activity of mammalian PI3K. Mammalian PI3K overproduced in yeast cells, by catalyzing the generation of $\mathrm{PIP}_{3}$ at the expense of $\mathrm{PIP}_{2}$ (which is essential for yeast growth), leads to $\mathrm{PIP}_{2}$ depletion thereby inhibiting yeast growth [10]. Importantly, Vps34, a class III PI3K and the sole PI3K homolog in S. cerevisiae, catalyzes the conversion of phosphatidyl inositol (PI) into phosphatidyl inositol 3phosphate (PIP) but is unable to use $\mathrm{PIP}_{2}$ as a substrate $[16,17]$. Therefore the $\mathrm{PIP}_{2}$ depletion observed upon expression of mammalian PI3K in yeast directly reflects its kinase activity and cannot be imputed to an endogenous yeast PI3K activity. To detect and follow the fate of $\mathrm{PIP}_{2}$ in yeast, cells were co-transformed with a plasmid expressing a fluorescent $\mathrm{PIP}_{2}$-specific reporter consisting of GFP fused to two tandem copies of the $\mathrm{PH}$ domain of mammalian phospholipase $\delta 1$. In $W T$ yeast cells, this reporter fusion protein specifically decorates $\mathrm{PIP}_{2}$ in the plasma membrane [13]. As previously observed [10], we found that the reporter clearly and prominently stained the plasma membrane of all cells overexpressing p110 ${ }^{\mathrm{K} 802 \mathrm{R}}$, in the presence or absence of the various PUFA (Figure 4, right rows), similar to the staining of cells in which the expression of $\mathrm{p} 110 \alpha^{\mathrm{K} 802 \mathrm{R}}$ was not induced. This confirms the inability of $\mathrm{p} 110 \alpha^{\mathrm{K} 802 \mathrm{R}}$ to catalyze conversion of $\mathrm{PIP}_{2}$ to $\mathrm{PIP}_{3}$. In contrast, in cells in which $\mathrm{p} 110 \alpha$ was overproduced, only diffuse cytoplasmic fluorescence was observed, and this in about 95\% of the cells (Figure 4, left rows, second line), indicating substantial $\mathrm{PIP}_{2}$ depletion from the plasma membrane. This effect was specific: the reporter clearly and prominently stained the plasma membrane of all control cells in which the expression of p110 $\alpha$ was not induced (Figure 4, left rows, first line). Cells overproducing p110 $\alpha$ were treated with AA, EPA or DHA and tested for staining. Interestingly, we observed normal plasma membrane localization of the GFP signal in a significant proportion of cells $(23 \%, 30 \%$ and $28 \%$ respectively). This confirms that these various PUFA inhibited the kinase activity of mammalian PI3K in yeast, thereby counteracting the depletion of $\mathrm{PIP}_{2}$ (Figure 4, left rows, lines 3 to 5). As controls, we checked that the three PUFA had no effect on the plasma membrane localization of the marker in cells overexpressing p110 $\alpha^{\mathrm{K} 802 \mathrm{R}}$ (Figure 4, right rows, lines 3 to 5). 


\section{DISCUSSION}

Our results strongly suggest that in a yeast model reconstituting the PI3K/PTEN/Akt pathway, three long chain PUFA, namely AA, EPA and DHA, inhibited PI3K activity. This is in accordance with previous observations in vivo in liver and muscle of rats receiving a diet enriched with a small amount of fish oils (rich in EPA and DHA) [8]. In this study, the PI3K activity was evaluated in vitro after immunoprecipitation of tyrosine phosphorylated proteins, thereby justifying the use, in the present study, of the yeast-based system where PI3K activity is directly correlated to in vivo measurable phenotypes: growth inhibition and depletion of $\mathrm{PIP}_{2}$ from the plasma membrane. The novel finding of our study is, therefore, that the inhibition of PI3K activity by AA, EPA and DHA may be direct, like the effects of LY294002 or wortmannin. There are several arguments for a direct mechanism of inhibition. The inhibitory effect previously observed in vivo in rats [8] was reproduced in our exogenous (for example the plasma membrane composition differs between yeast and mammalian cells and, yeast cells have a cell wall) and simplified model, and this is a strong argument in favor of a direct inhibition. Indeed, the PI3K/PTEN/Akt pathway was reconstituted in yeast, so the inhibitory effect of AA, EPA and DHA on PI3K activity was independent of any association with most of the other molecules belonging to this pathway, such as insulin receptor substrates 1 or 2 (IRS-1 or IRS-2), two upstream factors that regulate type 1A PI3K activity in vivo in mammals [18]. It was also not due to an indirect effect on the level of expression of the PI3K gene. Indeed, none of the three PUFA altered the steady-state abundance of PI3K or alleviated growth inhibition due to hof $1 \triangle \mathrm{PEST}$ overexpression from the same galactose-inducible GAL1 promoter. It is also unlikely that the inhibitory effect of the three long chain PUFA was due to one or more of their metabolites because the metabolism of long chain n-3 PUFA and of AA generate different metabolites. The inhibitory effect also cannot be explained by inhibition of palmitoylation of PI3K because 2-bromopalmitate which inhibits palmitoylation [19] did not inhibit PI3K.

AA, EPA and DHA (fatty acids with chain length $\geq 20$ carbons and with 4,5 and 6 double bonds respectively) had similar inhibitory effects on PI3K activity whereas oleic acid (chain length: 18 carbons and one double bond) had no such inhibitory effect. This implicates chain length, i.e. $\geq 20 \mathrm{C}$, and/or the high degree of unsaturation as possible determinants of the inhibitory effect of the three PUFA on PI3K activity. 
However, we did not address the exact mechanisms underlying the inhibitory effect of long chain PUFA on

PI3K. LY294002 and wortmannin, the most widely used PI3K inhibitors, both competitively target the ATP/substrate-binding site of the p110 catalytic subunit [3]. Further studies are needed to determine whether the inhibitory effect of AA, EPA and DHA involves this type of mechanism. Note that EPA and DHA are potent non competitive inhibitors of several protein kinases, including pKA, pKC, MAPK and CaMKII [7].

PI3K/PTEN signaling is central to mechanisms of tumorigenesis, angionesis, and anti-cancer drug and radio resistance [2, 6, 20-29]. Our observation that long chain n-3 and n-6 PUFA are natural PI3K inhibitors is therefore of particular interest. Indeed, there is diverse evidence suggesting that, in vitro or during nutritional interventions, long chain n-3 fatty acids enhance the response of tumors to the effects of anti-neoplastic agents (reviewed in [30]).

\section{ACKNOWLEDGMENTS}

Most of this work was done in the INSERM U613 laboratory of Claude Ferec who we thank for his continuous support and friendship. We thank P. Lehn and Y. Bizais for their warm welcome, encouragements and helpful scientific discussions. We are indebted to Maria Molina (Faculdad de Farmacia, Universidad Complutense de Madrid) for the kind gift of plasmids YCpLG-PI3K, YCpLG-PI3K ${ }^{\mathrm{K} 802 \mathrm{R}}$ and pRS426-GFP-2xPH (PLC $)$ ). Thanks are also due to N. Desban for excellent technical assistance and to C. Voisset for critical reading of the manuscript. This work was supported by the following grants: ANR "Maladies Rares" from the French government and AFM research grant to MB and EC, and AFM postdoctoral fellowship contract to EC.

\section{REFERENCES}

[1] Cantley, L. C., The phosphoinositide 3-kinase pathway. Science. 2002, 296, 1655-7.

[2] Carnero, A., Blanco-Aparicio, C., Renner, O., Link, W., et al., The PTEN/PI3K/AKT signalling pathway in cancer, therapeutic implications. Curr Cancer Drug Targets. 2008, 8, 187-98.

[3] Marone, R., Cmiljanovic, V., Giese, B. and Wymann, M. P., Targeting phosphoinositide 3-kinase: moving towards therapy. Biochim Biophys Acta. 2008, 1784, 159-85.

[4] Katso, R., Okkenhaug, K., Ahmadi, K., White, S., et al., Cellular function of phosphoinositide 3-kinases: implications for development, homeostasis, and cancer. Annu Rev Cell Dev Biol. 2001, 17, 615-75.

[5] Vivanco, I. and Sawyers, C. L., The phosphatidylinositol 3-Kinase AKT pathway in human cancer. Nat Rev Cancer. 2002, 2, 489-501. 
[6] Bussink, J., van der Kogel, A. J. and Kaanders, J. H., Activation of the PI3-K/AKT pathway and implications for radioresistance mechanisms in head and neck cancer. Lancet Oncol. 2008, 9, 288-96.

[7] Mirnikjoo, B., Brown, S. E., Kim, H. F., Marangell, L. B., et al., Protein kinase inhibition by omega-3 fatty acids. J Biol Chem. 2001, 276, 10888-96.

[8] Corporeau, C., Foll, C. L., Taouis, M., Gouygou, J. P., et al., Adipose tissue compensates for defect of phosphatidylinositol 3'-kinase induced in liver and muscle by dietary fish oil in fed rats. Am J Physiol Endocrinol Metab. 2006, 290, E78-E86.

[9] DeGraffenried, L. A., Friedrichs, W. E., Fulcher, L., Fernandes, G., et al., Eicosapentaenoic acid restores tamoxifen sensitivity in breast cancer cells with high Akt activity. Ann Oncol. 2003, 14, 1051-6.

[10] Rodriguez-Escudero, I., Roelants, F. M., Thorner, J., Nombela, C., et al., Reconstitution of the mammalian PI3K/PTEN/Akt pathway in yeast. Biochem J. 2005, 390, 613-23.

[11] Blondel, M., Bach, S., Bamps, S., Dobbelaere, J., et al., Degradation of Hof1 by SCF(Grr1) is important for actomyosin contraction during cytokinesis in yeast. Embo J. 2005, 24, 1440-52.

[12] Guthrie, C. and Fink, G. R., Guide to Yeast Genetics and Molecular Biology. Academic Press Inc. 1991, San Diego, CA.

[13] Stefan, C. J., Audhya, A. and Emr, S. D., The yeast synaptojanin-like proteins control the cellular distribution of phosphatidylinositol (4,5)-bisphosphate. Mol Biol Cell. 2002, 13, 542-57.

[14] Bach, S., Talarek, N., Andrieu, T., Vierfond, J. M., et al., Isolation of drugs active against mammalian prions using a yeast-based screening assay. Nat Biotechnol. 2003, 21, 1075-81.

[15] Bach, S., Tribouillard, D., Talarek, N., Desban, N., et al., A yeast-based assay to isolate drugs active against mammalian prions. Methods. 2006, 39, 72-7.

[16] Stack, J. H. and Emr, S. D., Vps34p required for yeast vacuolar protein sorting is a multiple specificity kinase that exhibits both protein kinase and phosphatidylinositol-specific PI 3-kinase activities. J Biol Chem. 1994, 269, 31552-62.

[17] Strahl, T. and Thorner, J., Synthesis and function of membrane phosphoinositides in budding yeast, Saccharomyces cerevisiae. Biochim Biophys Acta. 2007, 1771, 353-404.

[18] Kanzaki, M., Insulin receptor signals regulating GLUT4 translocation and actin dynamics. Endocr J. 2006, 53, 267-93.

[19] Webb, Y., Hermida-Matsumoto, L. and Resh, M. D., Inhibition of protein palmitoylation, raft localization, and $\mathrm{T}$ cell signaling by 2-bromopalmitate and polyunsaturated fatty acids. J Biol Chem. 2000, 275, 261-70.

[20] Lindsley, C. W., Barnett, S. F., Layton, M. E. and Bilodeau, M. T., The PI3K/Akt pathway: recent progress in the development of ATP-competitive and allosteric Akt kinase inhibitors. Curr Cancer Drug Targets. 2008, 8, 7-18.

[21] Juntilla, M. M. and Koretzky, G. A., Critical roles of the PI3K/Akt signaling pathway in T cell development. Immunol Lett. 2008, 116, 104-10.

[22] Maira, S. M., Voliva, C. and Garcia-Echeverria, C., Class IA phosphatidylinositol 3-kinase: from their biologic implication in human cancers to drug discovery. Expert Opin Ther Targets. 2008, 12, 223-38.

[23] Haluska, F., Pemberton, T., Ibrahim, N. and Kalinsky, K., The RTK/RAS/BRAF/PI3K pathways in melanoma: biology, small molecule inhibitors, and potential applications. Semin Oncol. 2007, 34, 546-54.

[24] Wee, S., Lengauer, C. and Wiederschain, D., Class IA phosphoinositide 3-kinase isoforms and human tumorigenesis: implications for cancer drug discovery and development. Curr Opin Oncol. 2008, 20, 77-82.

[25] Jiang, B. H. and Liu, L. Z., PI3K/PTEN signaling in tumorigenesis and angiogenesis. Biochim Biophys Acta. 2008, 1784, 150-8.

[26] Engelman, J. A., The role of phosphoinositide 3-kinase pathway inhibitors in the treatment of lung cancer. Clin Cancer Res. 2007, 13, s4637-40.

[27] Kumar, A. and Carrera, A. C., New functions for PI3K in the control of cell division. Cell Cycle. 2007, 6, 1696-8.

[28] Cantley, L. C., The role of phosphoinositide 3-kinase in human disease. Harvey Lect. 2004, 100, 103 22.

[29] McCubrey, J. A., Steelman, L. S., Chappell, W. H., Abrams, S. L., et al., Roles of the Raf/MEK/ERK pathway in cell growth, malignant transformation and drug resistance. Biochim Biophys Acta. 2007, 1773, 1263-84. 
Couplan et al. $\quad$ Inhibition of PI3K by fatty acids

[30] Pardini, R. S., Nutritional intervention with omega-3 fatty acids enhances tumor response to antineoplastic agents. Chem Biol Interact. 2006, 162, 89-105.

3

4

5

6

7

8

9

10

11

12

13

14

15

16

17

18

19

20

21

22

23

24

25

26

27

28

29

30

31

32

33

34

35

36

37

38

39

40

41

42

43

44

45

46

47

48

49

50

51

52

53

54

55

56

57

58

59 


\section{FIGURE LEGENDS}

\section{Figure 1: flowchart of the mammalian p110 overexpression-driven toxicity in yeast}

A WT yeast strain was transformed with a vector allowing expression from the strong galactoseinducible GAL1 promoter, of either $\mathrm{p} 110 \alpha$, the catalytic subunit of PI3K (upper panel) or p110 ${ }^{\mathrm{K} 802 \mathrm{R}}$, a catalytically inactive form of PI3K (lower panel). Both proteins are fused in frame at their C-termini with the -CAAX box of H-ras which targets them to the plasma membrane to compensate for the absence of $\mathrm{p} 85$, the PI3K regulatory subunit which targets $\mathrm{p} 110 \alpha$ to the plasma membrane in its normal context in mammals. Galactose (right diagram) but not glucose (repressing conditions, left diagram) activates transcription from the strong $G A L 1$ promoter therefore allowing a high level of expression of p110 $\alpha$ which in turn converts the essential PIP $_{2}$ into PIP $_{3}$ (upper panel). As a consequence, yeast cell growth is strongly impaired due to altered morphogenesis and vesicular trafficking. p110 ${ }^{\mathrm{K} 802 \mathrm{R}}$, even when overexpressed at a similar level, has an effect neither on yeast cells growth nor on $\mathrm{PIP}_{2}$ level (lower panel) showing that this growth inhibition effect involves the kinase activity of PI3K.

Figure 2: toxicity linked to overexpression of the mammalian p110 $\alpha$ catalytic subunit of PI3K in yeast is alleviated by several fatty acids

WT yeast strain (K699) expressing either p110 , the catalytic subunit of PI3K (left Petri plates) or $\mathrm{p} 110 \alpha^{\mathrm{K} 802 \mathrm{R}}$, a catalytically inactive form of PI3K (right Petri plates), was spread on selective media containing either glucose (lower plates) which inhibits expression from the galactose-inducible GAL1 promoter or galactose (upper plates) which allows a strong expression from the promoter and thus of $\mathrm{p} 110 \alpha$ (left row) or $\mathrm{p} 110 \alpha^{\mathrm{K} 802 \mathrm{R}}$ (right row). Sterile filters (similar to those used for antibiograms) were then placed on the agar surface and the various compounds to be tested were spotted onto the filters as indicated in the scheme on the left. Pictures of the Petri dishes after 4 days of incubation at $30^{\circ} \mathrm{C}$ are shown. Only p110 $\alpha$ expression led to a profound growth defect, as previously shown. AA, EPA and 
DHA alleviated this growth defect to an even greater extent than LY294002, the positive control (a known chemical inhibitor of PI3K).

\section{Figure 3: the ability of fatty acids -AA, EPA and DHA- to alleviate PI3K-driven toxicity in yeast is not}

\section{due to an effect on GAL1 promoter-driven PI3K expression}

a. An experiment similar to that described in Figure $\mathbf{2}$ was performed except that the yeast cells used were K699 hof14 transformed either with a vector allowing strong expression of hof1 $\mathrm{PEST}$ (left Petri plate) from the same strong galactose-inducible GAL1 promoter as used for PI3K overexpression or, with an empty vector as a control (right Petri dish). As previously reported [11] when overexpressed, hof $1 \triangle \mathrm{PEST}$ led to a strong growth defect (compare left plate with right plate) because it blocks cytokinesis. Note that none of the tested compounds (indicated in the schema on the left) alleviated this growth defect. Therefore, their effect of alleviating toxicity induced by overexpression of $\mathrm{p} 110 \alpha$ is not due to their interference with the GAL1 promoter-driven overexpression.

b. WT yeast strain (K699) transformed either with an empty vector (lane 1) or with plasmid YCpLGPI3K allowing overexpression of $\mathrm{p} 110 \alpha$, the catalytic subunit of PI3K (lanes 2-6) was grown in the presence or absence of galactose and of $0.005 \%$ of the fatty acids as indicated. Cell extracts were prepared and analyzed by Western blot using an antibody raised against p110 (upper gel) or actin (lower gel) as a loading control. None of the tested fatty acids tested had a significant effect on the steady state abundance of $\mathrm{p} 110 \alpha$ indicating that their ability to alleviate toxicity due to overexpression of $\mathrm{p} 110 \alpha$ is not caused by an effect on the amount of this catalytic subunit of PI3K.

c. Left curves: growth curves of WT yeast strain (K699) overexpressing either p110 $\alpha$ (PI3K, gray filled circles) or $\mathrm{p} 110 \alpha^{\mathrm{K} 802 \mathrm{R}}$ (PI3K-K802R, black circles). Overexpression of the two forms of PI3K was induced at time 0 by addition of galactose. Overexpression of $\mathrm{p} 110 \alpha$ strongly inhibited yeast growth and this effect was first evident $6 \mathrm{H}$ after induction of overexpression. Right curves: WT yeast strain (K699) overexpressing p110 $\alpha$ was treated either with DMSO (gray-filled triangles) or with $0.005 \%$ 
DHA (black diamonds). DHA partially suppressed the ability of overexpressed p110 $\alpha$ to inhibit yeast growth.

\section{Figure 4: AA, EPA and DHA lead to a partial suppression of $\mathrm{PIP}_{2}$ depletion}

WT yeast strain (K699) was transformed with a vector expressing GFP-2xPH, a fluorescent $\mathrm{PIP}_{2}$-specific reporter together with either YCpLG-PI3K allowing overexpression of p110 $\alpha$ (PI3K, left rows) or YCpLG-PI3K $\mathrm{K}^{\mathrm{K} 802 \mathrm{R}}$ allowing overexpression of $\mathrm{p} 110 \alpha^{\mathrm{K} 802 \mathrm{R}}$ (PI3K $\mathrm{K}^{\mathrm{K} 802 \mathrm{R}}$, right rows). These strains were grown in a liquid selective medium in the absence (first line) or in the presence of galactose (lines 2 to 5) as indicated on the left $\left(P_{G A L 1}\right.$ OFF or ON) to repress or induce, respectively, expression of p110 $\alpha$ or $\mathrm{p} 110 \alpha^{\mathrm{K} 802 \mathrm{R}}$. Under repressing conditions, GFP staining and therefore $\mathrm{PIP}_{2}$ was localized at the plasma membrane (first line). As previously observed [10], only overexpression of PI3K/ p110 $\alpha$ led to the depletion of $\mathrm{PIP}_{2}$ from the plasma membrane in almost all cells (only $5 \%$ of the cells maintained a GFP signal at the membrane, left rows, second line), whereas $100 \%$ of cells overexpressing PI3K $\mathrm{K}^{\mathrm{K} 802 \mathrm{R}}$ maintained GFP signal at the plasma membrane (right rows, second line). The effect of AA (third line), EPA (fourth line) and DHA (fifth line) on $\mathrm{PIP}_{2}$ depletion was evaluated. The presence of each of these fatty acids led to a strong increase in the percentage of cells exhibiting a GFP signal at the plasma membrane (left rows, lines 3 to 5), indicating a reduced $\mathrm{PIP}_{2}$ depletion (an thus reduced PI3K activity), whereas they had no effect on the GFP signal at the membrane in cells overexpressing PI3K ${ }^{\mathrm{K} 802 \mathrm{R}}$ (right rows, lines 3 to 5). 
Figure 1
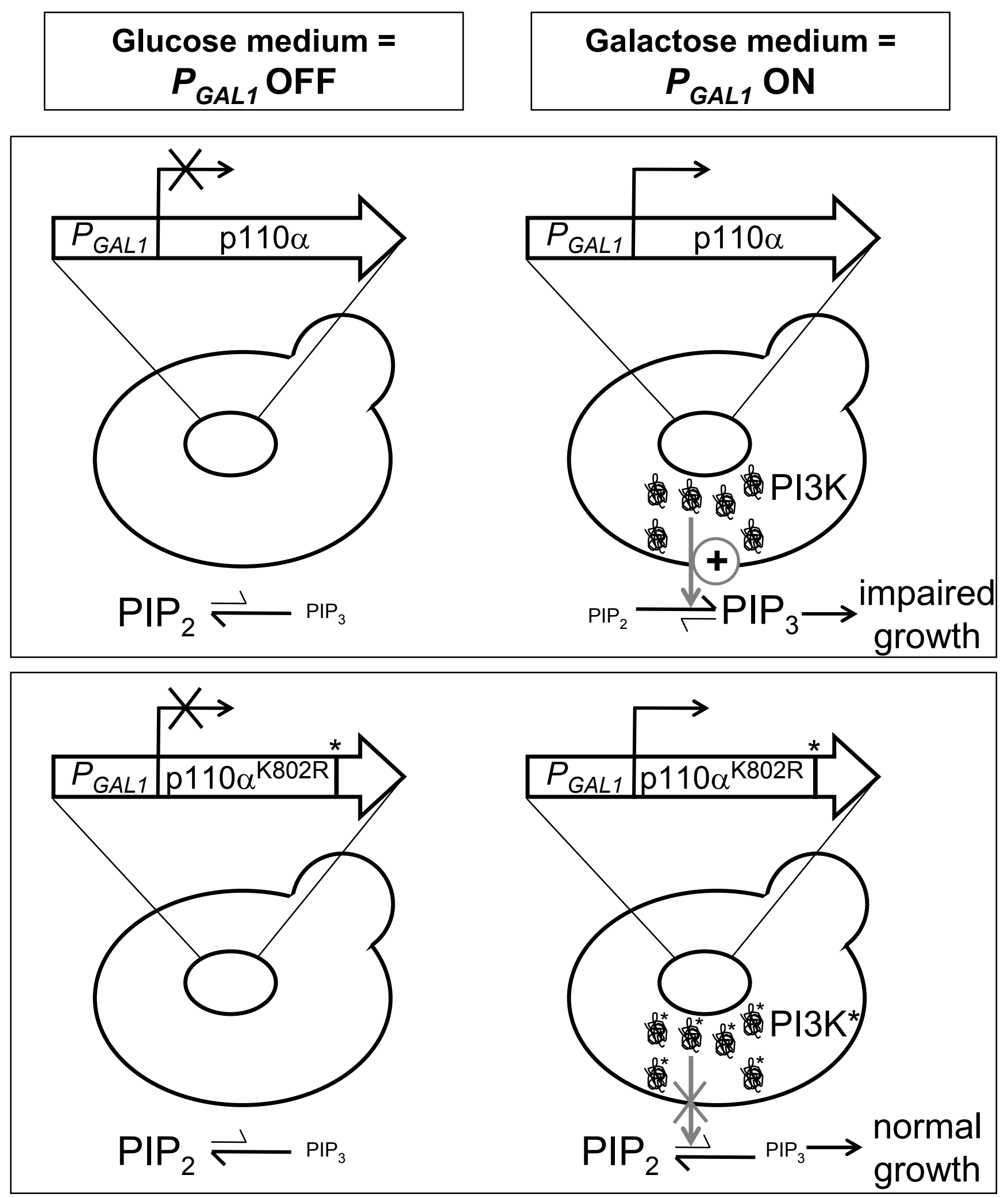

p110 $\alpha=$ PI3K catalytic subunit 
Figure 2
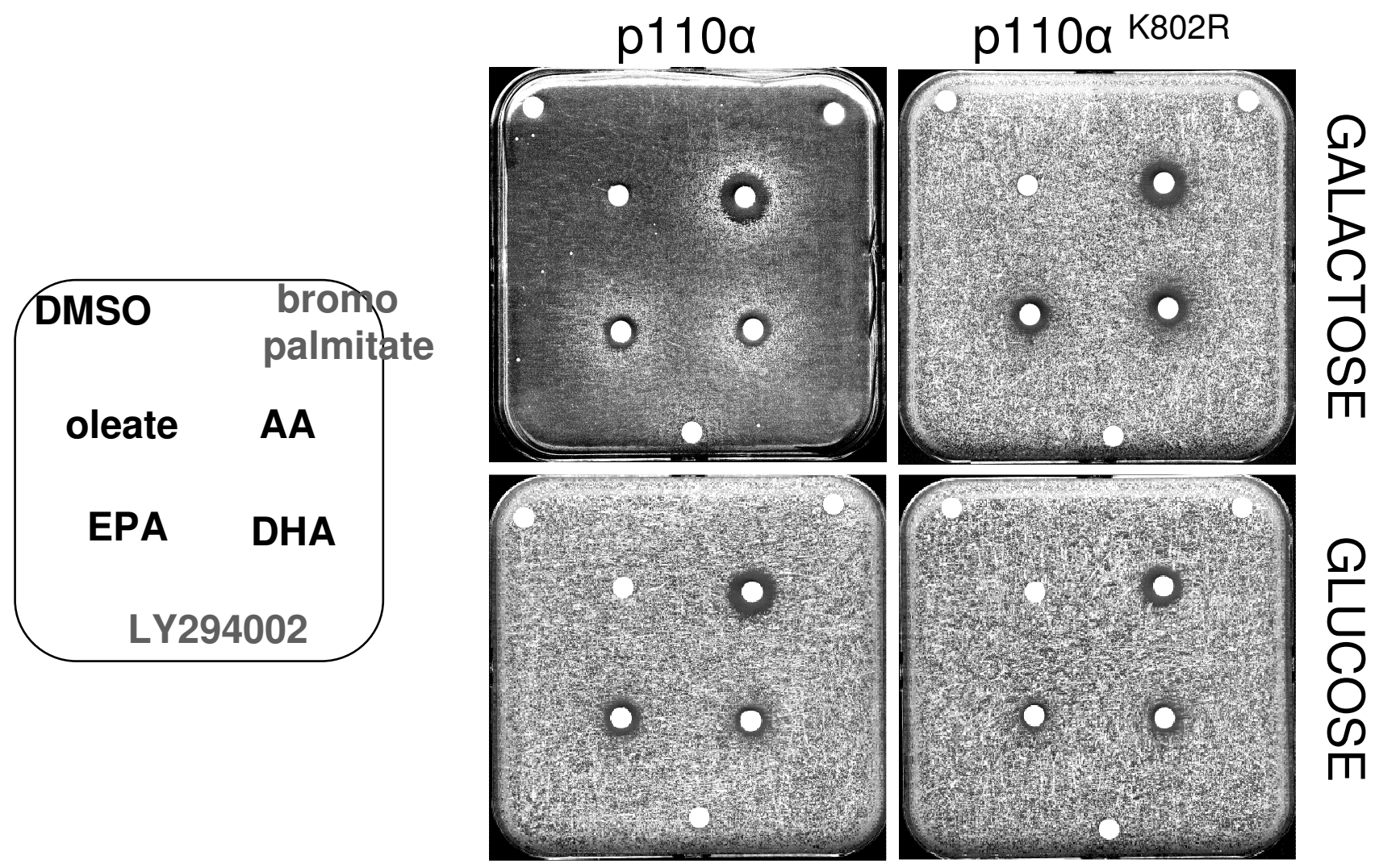

$\Omega$
\ulcorner
$\complement$
0
0
ח 


\section{Figure 3}

a
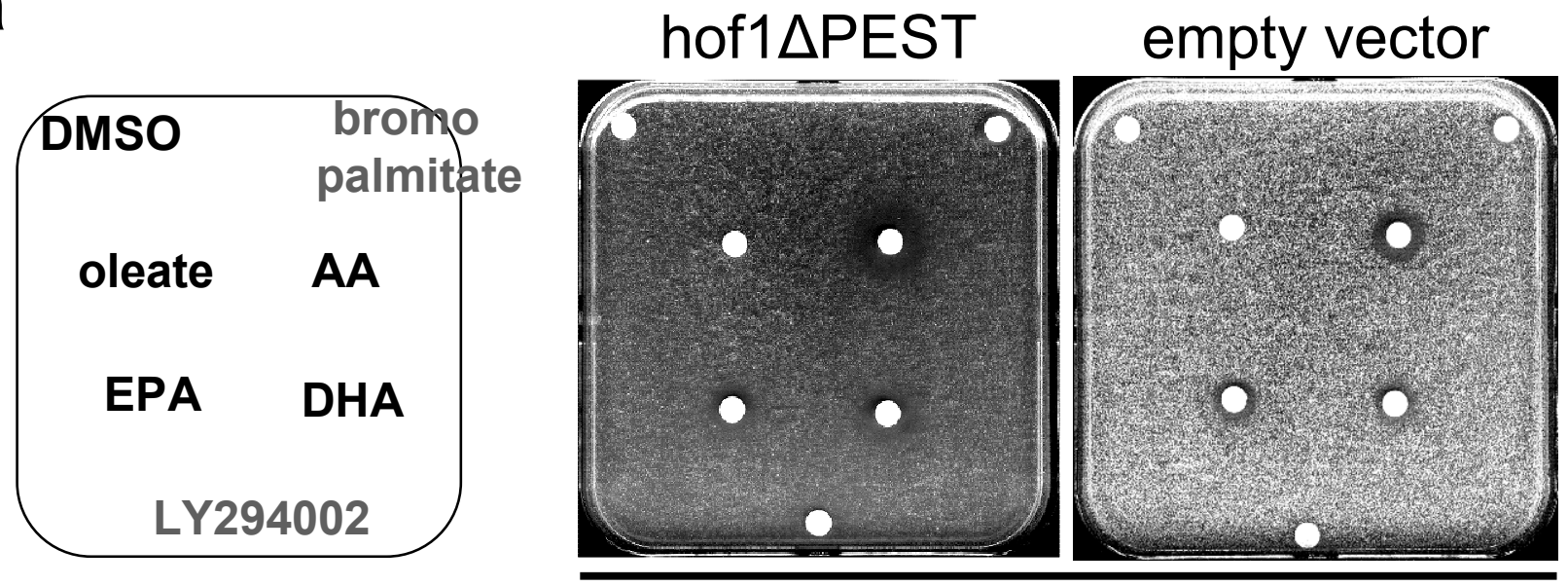

GALACTOSE

b

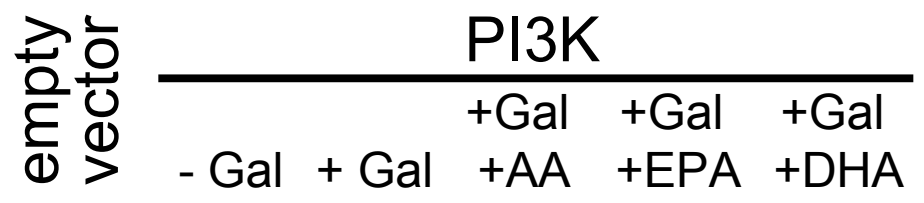

C

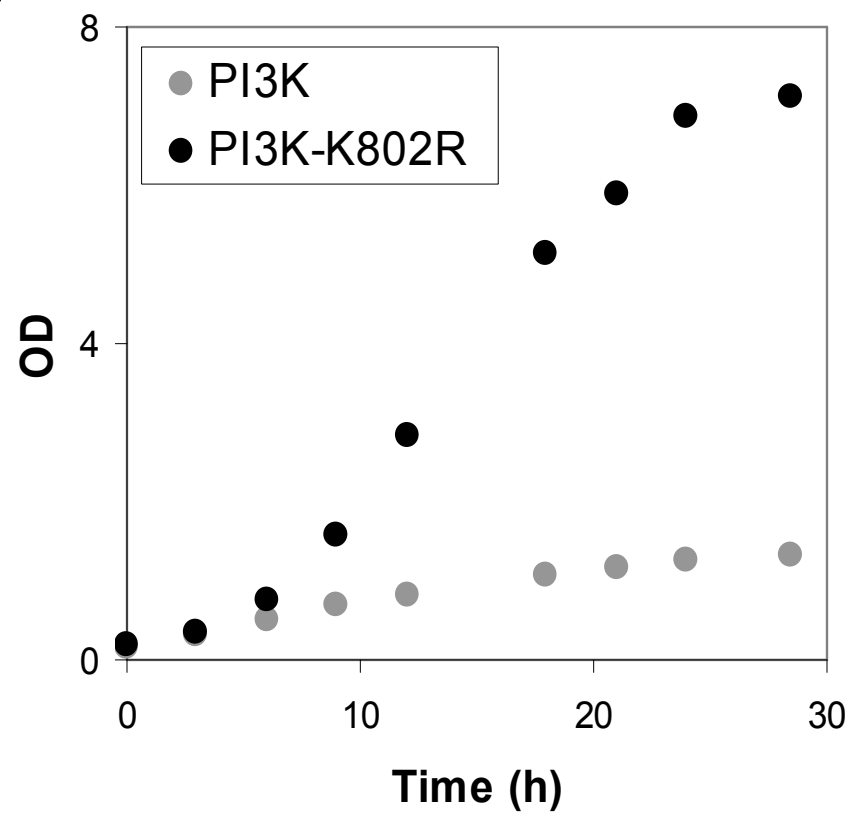

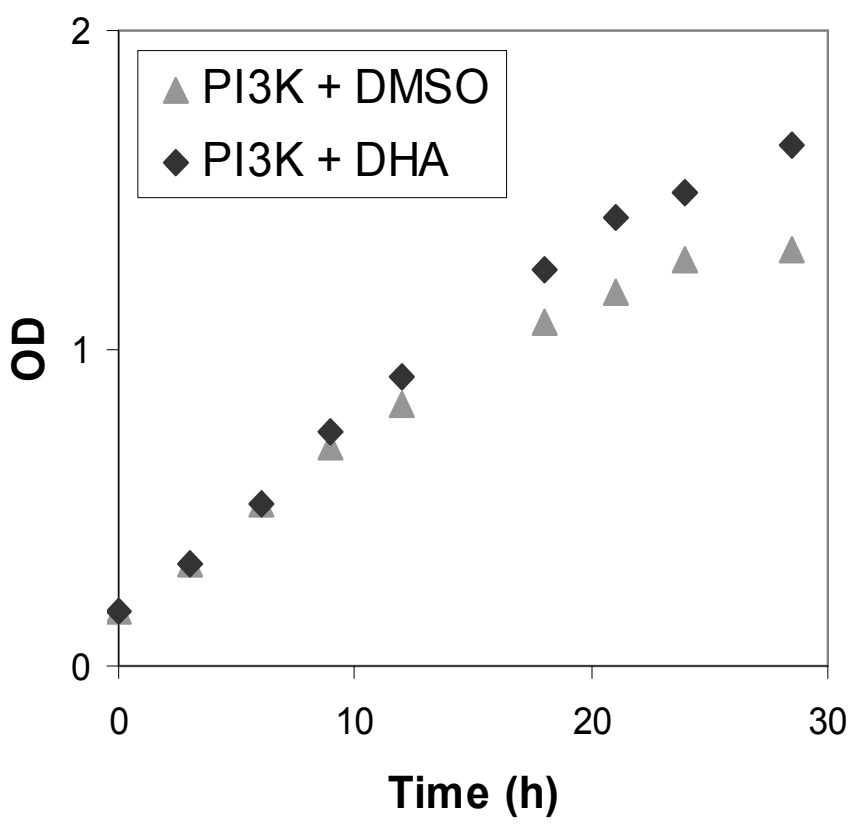


Figure 4

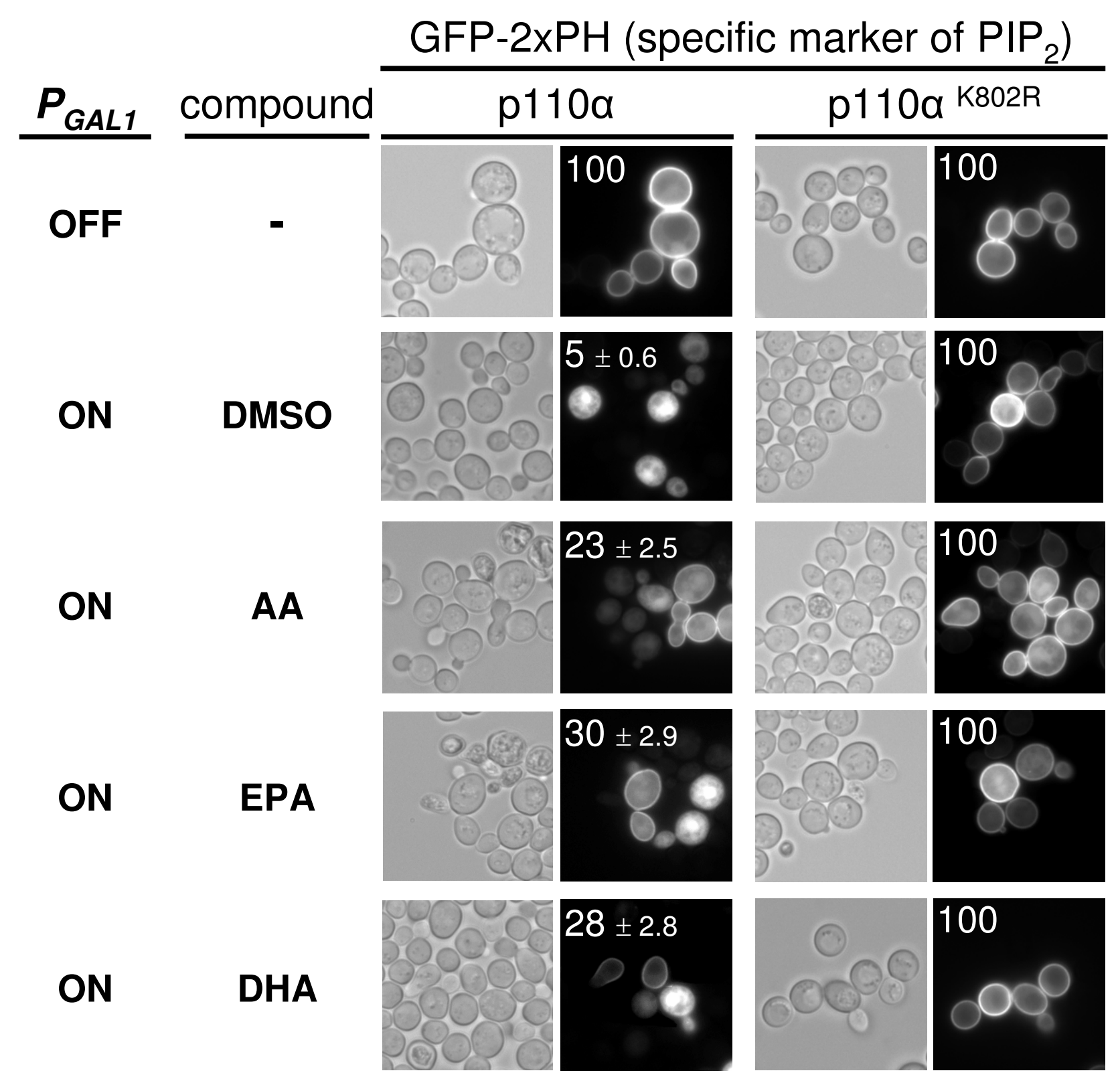

Numbers indicate the percentage of cells with GFP signal at the membrane 